\title{
Clinical and Microbiological Outcomes of Ceftazidime-Avibactam Treatment in Adults with Gram-Negative Bacteremia: A Subset Analysis from the Phase 3 Clinical Trial Program
}

\author{
John E. Mazuski · Florian Wagenlehner (D) - Antoni Torres (D) • \\ Yehuda Carmeli · Joseph W. Chow · Dalia Wajsbrot · Gregory G. Stone • \\ Paurus Irani · David Bharucha $\cdot$ Karen Cheng (D) · Margaret Tawadrous (D)
}

Received: December 23, 2020 / Accepted: July 16, 2021 / Published online: August 10, 2021

(C) Pfizer 2021

\section{ABSTRACT}

Introduction: This exploratory analysis assessed efficacy and safety outcomes in patients with Gram-negative bacteremia treated with ceftazidime-avibactam or comparator across five phase 3, randomized, controlled, multi-center

Supplementary Information The online version contains supplementary material available at https://doi. org/10.1007/s40121-021-00506-7.

J. E. Mazuski

Department of Surgery, Washington University

School of Medicine, St Louis, MO, USA

F. Wagenlehner

Department of Urology, Pediatric Urology and

Andrology, Justus-Liebig-University, Giessen,

Germany

A. Torres

Department of Pulmonology, Hospital Clinic, University of Barcelona, IDIBAPS, CIBERES, ICREA, Barcelona, Spain

Y. Carmeli

Division of Epidemiology, National Institute for Antibiotic Resistance and Infection Control, Tel Aviv Sourasky Medical Center, Tel Aviv, Israel

J. W. Chow

Global Product Development, Pfizer, Collegeville,

PA, USA

D. Wajsbrot

Biostatistics, Pfizer, New York, NY, USA trials in adults with complicated intra-abdominal infection (cIAI), complicated urinary tract infection (cUTI)/pyelonephritis, hospital-acquired pneumonia (HAP), and ventilator-associated pneumonia (VAP).

Methods: In each trial, RECLAIM and RECLAIM 3 (CIAI; NCT01499290/NCT01726023), REPRISE (cIAI/cUTI; NCT01644643), RECAPTURE (cUTI; NCT01595438/NCT01599806), and REPROVE (HAP/VAP; NCT01808092), patients were ran-

\section{G. G. Stone}

Microbiology, Internal Medicine, Pfizer, Groton, CT, USA

P. Irani

Global Medical Affairs, Pfizer, Tadworth, Surrey, UK

D. Bharucha

Clinical Development, AbbVie, Madison, NJ, USA

K. Cheng

Safety Surveillance and Risk Management, Pfizer,

Sandwich, Kent, UK

M. Tawadrous $(\bowtie)$

Global Product Development, Pfizer, Inc, 445 Eastern

Point Road, Groton, CT 06340, USA

e-mail: Margaret.Tawadrous@pfizer.com 
domized $1: 1$ to intravenous ceftazidime-avibactam (plus metronidazole for those with cIAI) or comparators (carbapenems in $>97 \%$ patients) for 5-21 days. Efficacy assessments included clinical and microbiological responses at the test-of-cure visit in the pooled Gramnegative extended microbiologically evaluable (GNeME) population (bacteremia subset). Safety outcomes were summarized for patients with positive bacterial blood culture(s) at baseline who received $\geq 1$ dose of study treatment.

Results: The overall safety population included 4050 patients (ceftazidime-avibactam, $n=2024$; comparator, $n=2026$ ). The GNeME population (bacteremia subset) comprised 101 patients (ceftazidime-avibactam, $n=54$; comparator, $n=47$ ). Clinical cure rates (all indications combined) were $47 / 54(87.0 \%)$ for ceftazidimeavibactam and 39/47 (83.0\%) for comparators; favorable microbiological response rates were $43 / 54(79.6 \%)$ and 32/47 (68.1\%), respectively. Clinical and microbiological responses in the bacteremia subset were generally similar to those in the overall set. The pattern of adverse events in patients with bacteremia was similar between treatment groups and was consistent with the known safety profile of ceftazidimeavibactam.

Conclusion: This analysis provides supportive evidence of the efficacy and safety of ceftazidime-avibactam in patients with Gramnegative bacteremia associated with cIAI, cUTI/ pyelonephritis, or HAP/VAP.

Keywords: Bacteremia; Ceftazidime-avibactam; Complicated intra-abdominal infection; Complicated urinary tract infection; Efficacy; Hospital-acquired pneumonia

\section{Key Summary Points}

\section{Why carry out this study?}

Bacteremia associated with infection can result in substantial morbidity and mortality.

This exploratory analysis assessed efficacy and safety outcomes in patients with Gram-negative bacteremia treated with ceftazidime-avibactam or comparator across five phase 3 , randomized, controlled, multi-center trials in adults with complicated intra-abdominal infection (cIAI), complicated urinary tract infection (cUTI)/pyelonephritis, hospitalacquired pneumonia (HAP), and ventilator-associated pneumonia (VAP).

\section{What was learned from the study?}

Clinical and microbiological responses in the bacteremia subset were generally similar to those in the overall set.

The pattern of adverse events in patients with Gram-negative bacteremia was similar between treatment groups and consistent with the known safety profile of ceftazidime-avibactam.

This analysis provides supportive evidence of the efficacy and safety of ceftazidimeavibactam in patients with Gram-negative bacteremia associated with cIAI, cUTI/ pyelonephritis, or HAP/VAP.

\section{INTRODUCTION}

Bacteremia (the isolation of bacterial pathogens from blood culture) can arise either as a primary bloodstream infection or secondary to acute systemic infections [1], and when associated with infection can result in substantial morbidity and mortality [2]. Most cases of secondary bacteremia originate from lower respiratory tract and intra-abdominal and 
urinary tract infections, while ventilator-associated pneumonia (VAP) is a common source of infection in the intensive care unit $[3,4]$.

Gram-negative species, including Enterobacterales and Pseudomonas aeruginosa, are frequently involved in serious infections in hospital and healthcare settings and are capable of expressing multiple antibiotic resistance mechanisms [2].

Multidrug-resistant (MDR) Gram-negative bacteremia represents a clinical challenge due to a lack of guidance on optimal duration of treatment as well as the limited treatment options available $[5,6]$. As time to appropriate antibiotic therapy is a predictor of mortality in patients with MDR Gram-negative bacteremia, early appropriate treatment is vital for outcome optimization [4, 7].

Ceftazidime-avibactam, a combination of the cephalosporin ceftazidime and the novel non- $\beta$-lactam $\beta$-lactamase inhibitor avibactam, has demonstrated clinical and microbiological efficacy against target pathogens in a range of serious infections, including those caused by
MDR and extended-spectrum $\beta$-lactamase (ESBL)-producing Gram-negative isolates [8-12]. Ceftazidime-avibactam has also demonstrated in vitro activity against ESBL-, AmpC-, and class A (Klebsiella pneumoniae carbapenemase), class C, and some class D (OXA-48) serine carbapenemase-producing Gram-negative bacteria, but not metallo-beta-lactamase (class B) producers [13-15].

The adult clinical trial program formed the basis of the approval of ceftazidime-avibactam in the USA and EU for the treatment of complicated intra-abdominal infections (cIAIs) in combination with metronidazole, complicated urinary tract infections (cUTIs)/pyelonephritis, and hospital-acquired pneumonia (HAP; also referred to as nosocomial pneumonia [NP]), including VAP, including for cases of bacteremia associated with these infections [16-18]. Ceftazidime-avibactam is also approved in the EU for the treatment of infections caused by aerobic Gram-negative organisms in adult patients with limited treatment options [16, 18]. In 2019, the US label was
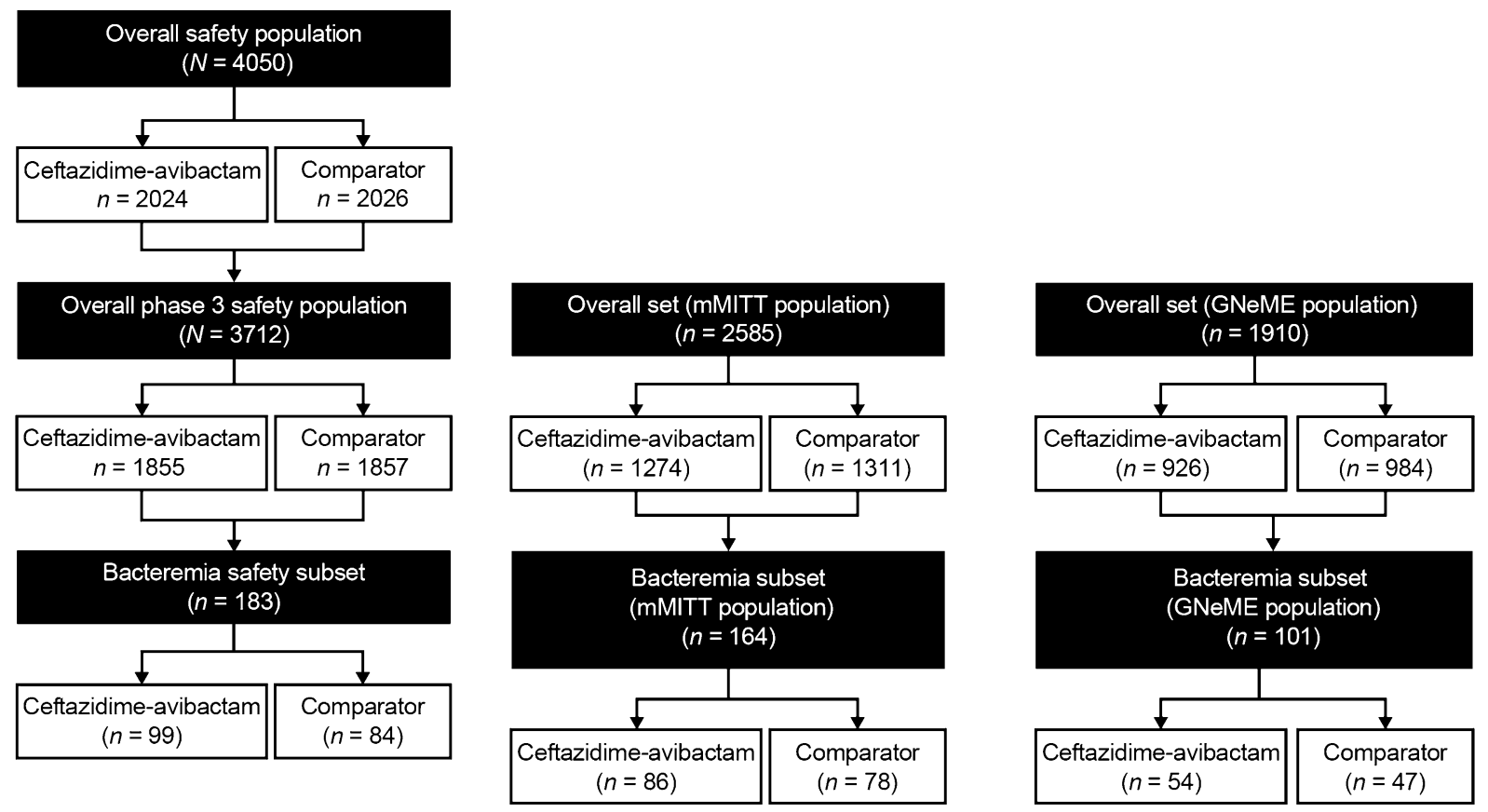

Fig. 1 Overview of analysis sets. GNeME Gram-negative extended microbiologically evaluable, $m M I T T$ microbiological modified intent to treat 


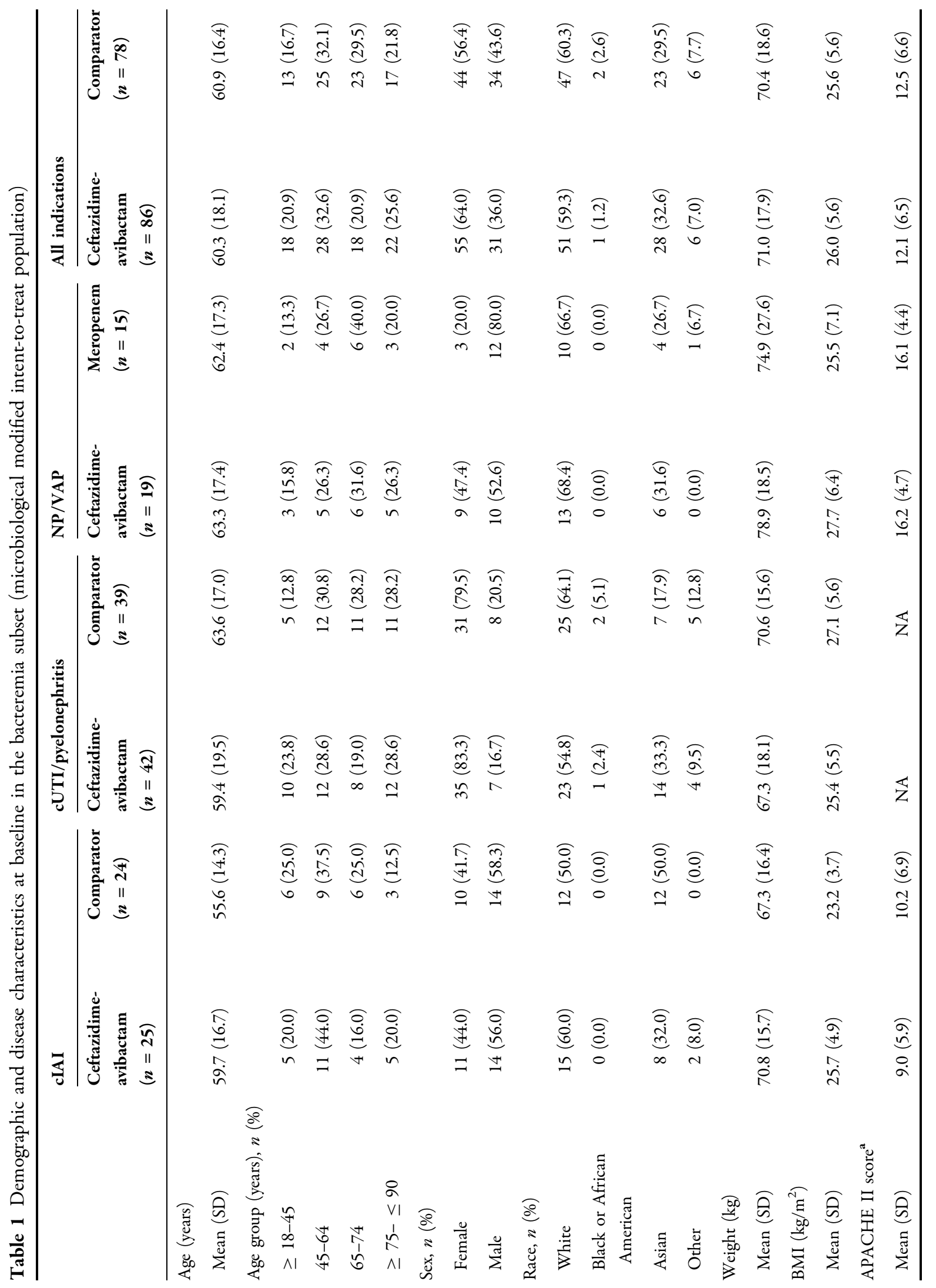




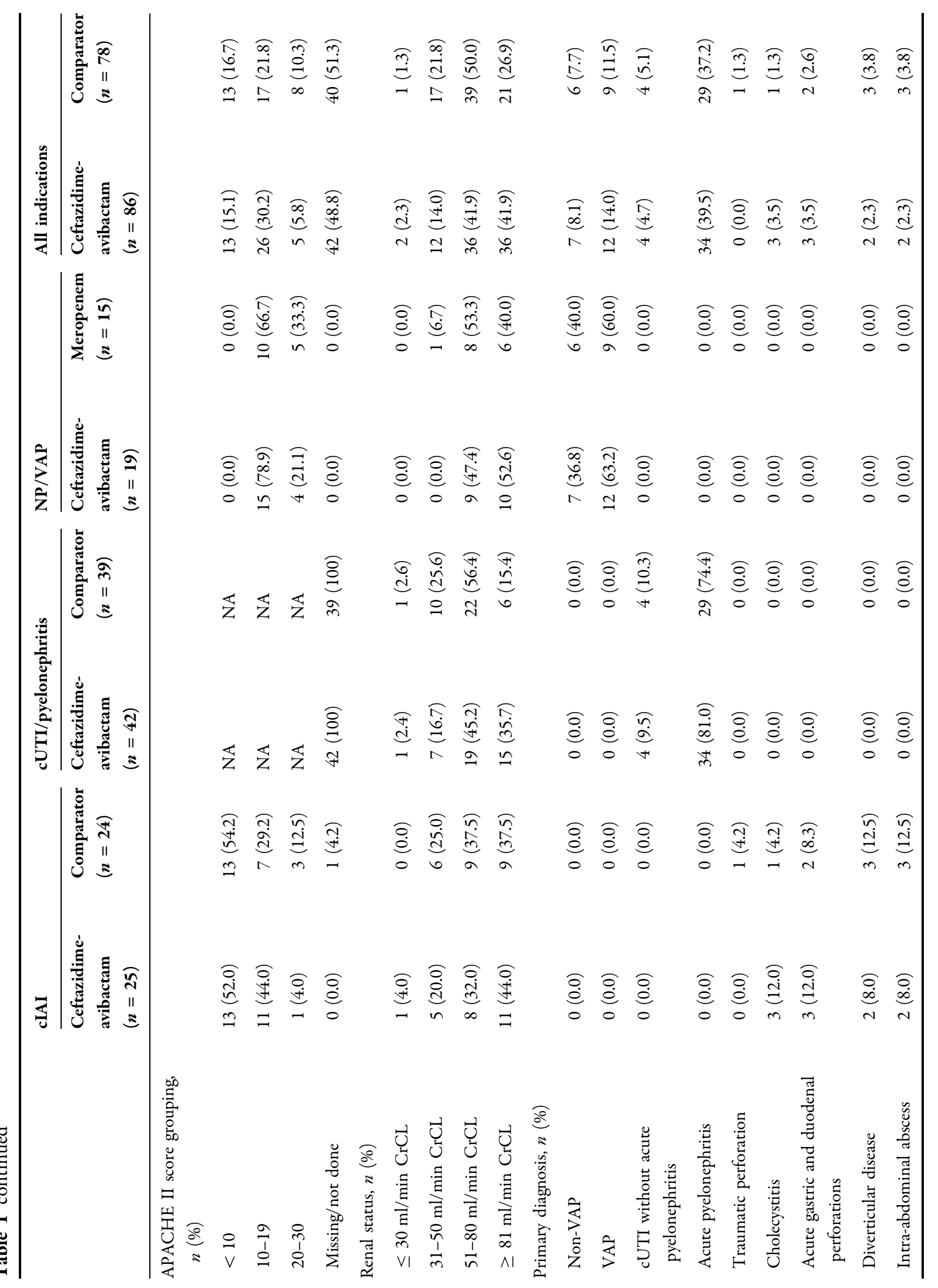




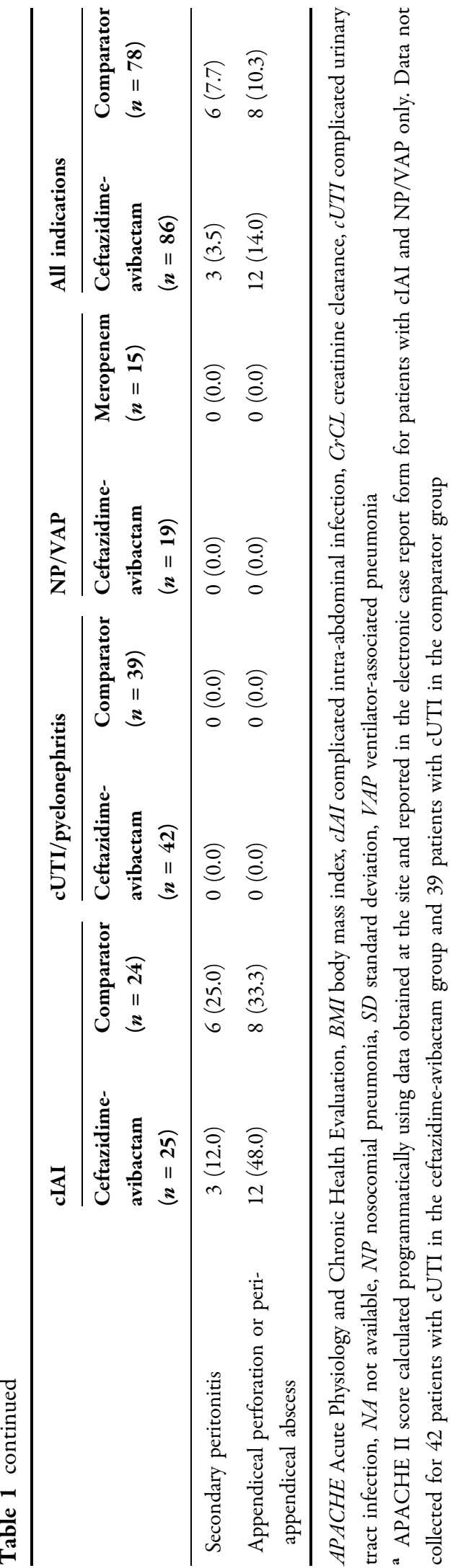

expanded to include children aged 3 months and older for the treatment of cIAI in combination with metronidazole and cUTI/ pyelonephritis [16]. The adult indications in the EU label have also recently been extended to include pediatric patients 3 months and older; this is the first European approval of an antibiotic to treat cUTI, cIAI, or HAP/VAP caused by resistant Gram-negative bacteria in children since at least 2011, when the European commission/World Health Organization agreed an "Action Plan against the rising threats from Antimicrobial Resistance" [17].

Safety data from two phase 2 and five phase 3 studies in the adult ceftazidime-avibactam clinical trial program $[8-12,19,20]$ were also evaluated in a recent pooled analysis (referred to here as the overall safety population) [21]. Each of the five phase 3 studies in the ceftazidime-avibactam clinical trial program included a small number of patients with bacteremia at baseline [8-12]. Data from the subgroup of patients with bacteremia from these studies have not previously been described.

As bacteremia is frequently a marker of more severe disease, subset analyses of patients with bacteremia may also provide additional supportive information on the use of ceftazidimeavibactam in patients with more serious Gramnegative infections. A descriptive analysis of pooled clinical trial data, discussed here, was undertaken to assess the clinical and microbiological outcomes and safety for the subset of patients with Gram-negative bacteremia across the ceftazidime-avibactam phase 3 clinical trials.

\section{METHODS}

\section{Ethics}

This subset analysis was based on five previously reported phase 3 ceftazidime-avibactam clinical trials. Ethics approval and patient consent to participate were obtained for each of the previously reported trials, and all trials were conducted in accordance with the Helsinki Declaration of 1964 and its later amendments. 


\section{Study Designs and Patient Populations}

An overview of the five phase 3 trials is provided in Table A1 (see supplementary online material). One study enrolled patients with NP: REPROVE (NCT01808092) [11]. Two studies enrolled patients with cUTI/pyelonephritis: RECAPTURE $1 / 2$ (NCT01595438 and NCT01599806; data from the two trials were analyzed as a single study) [12] and REPRISE (NCT01644643) [8], and three studies enrolled patients with cIAI: RECLAIM 1/2 (NCT01499290 and NCT01500239; data from the two clinical trials were analyzed as a single study) [9], RECLAIM 3 (NCT01726023) [10], and REPRISE [8].

Detailed methods and results for each study have been reported previously [8-12]. In brief, hospitalized patients with cIAI, cUTI/ pyelonephritis, or NP/VAP were randomized 1:1 to receive either ceftazidime-avibactam or comparator (which was a carbapenem in most cases) for 5-21 days. Ceftazidime-avibactam dosage for patients with creatinine clearance $>50 \mathrm{ml} / \mathrm{min}$ was $2000 / 500 \mathrm{mg}$ every $8 \mathrm{~h}$ (q8h) by 2 -h intravenous (i.v.) infusions. Patients with cIAI randomized to ceftazidimeavibactam treatment also received metronidazole $500 \mathrm{mg}$ (by 1-h i.v. infusion) q8h. Doses of ceftazidime-avibactam and comparators were adjusted for patients with renal impairment (creatinine clearance $<50 \mathrm{ml} / \mathrm{min}$ ). The comparator regimen in RECLAIM and REPROVE was meropenem $1000 \mathrm{mg}$ q8h (by 30-min i.v. infusion). In REPRISE and RECAPTURE, the comparator regimens were best available therapy (a carbapenem in $\sim 97 \%$ of cases) and doripenem $500 \mathrm{mg}$ q8h (by 30-min i.v. infusions), respectively.

Clinical cure was defined as complete resolution or significant improvement of signs and symptoms of the index infection such that no further antibacterial therapy, drainage, or surgical intervention was necessary. Favorable microbiological response included "eradication," defined as absence of the causative pathogen from appropriately obtained specimens at the site of infection, and "presumed eradication" (repeat cultures were not performed/clinically indicated in a patient who had a clinical response of cure). Efficacy assessments included clinical cure and favorable microbiological responses at the test-of-cure (TOC) visit ( 21-35 days after the last study treatment [RECLAIM, RECAPTURE, and REPROVE] or 7-10 days after the last infusion of study therapy [REPRISE]), and safety data were captured up to the late follow-up visit (28-52 days post-randomization).

\section{Post Hoc Pooling and Subset Analysis}

\section{Analysis Populations}

The overall safety population included the patients who received any dose of study drug treatment in the two phase 2 and five phase 3 ceftazidime-avibactam clinical studies.

Patients were categorized as having Gramnegative bacteremia at baseline if one or more study-qualifying pathogens were identified from a baseline blood culture. Confirmatory urine culture (of the same pathogen as detected in blood) was also required in RECAPTURE $1 / 2$.

For all efficacy and safety analysis bacteremia subsets, only patients from the phase 3 studies were included.

The microbiological modified intent-to-treat (mMITT) population included patients who received at least one dose of study drug, met the protocol-specific minimum disease criteria in each study, and had an etiological baseline pathogen isolated from the primary site of infection and/or blood, excluding those who were not expected to respond to either study treatment. Confirmatory urine culture (of the same pathogen as detected in blood) was also required in RECAPTURE $1 / 2$.

The Gram-negative extended microbiologically evaluable (GNeME) population was considered the primary analysis population; this was defined as a subset of the extended microbiologically evaluable population (i.e., patients who were protocol-compliant, had an evaluable clinical response, and had a study-qualifying baseline Gram-negative pathogen, regardless of susceptibility to study treatment), as specified in each individual study, limited to patients with aerobic Gram-negative bacteria isolated from the primary site of infection and/or blood. For 
the bacteremia subset, this population was further limited to individuals with Gram-negative pathogens isolated from the blood at baseline.

The bacteremia safety subset comprised patients from the five phase 3 studies who received any dose of study treatment and had Gram-negative bacteremia at baseline.

\section{Efficacy and Safety Analyses}

Efficacy outcomes of ceftazidime-avibactam and comparators were pooled across all studies by indication and for indications combined. Clinical and microbiological response rates at TOC were summarized for the bacteremia subset (GNeME and mMITT populations), and 95\% confidence intervals (CIs) for treatment differences were calculated using the unstratified Miettinen and Nurminen method [22]. Safety data (including adverse events [AEs] and clinical laboratory assessments) up to the last visit were summarized by indication and for all indications combined for the bacteremia safety subset. There were no formal statistical hypotheses for these analyses; all data were summarized descriptively.

\section{RESULTS}

\section{Analysis Populations}

The overall safety population for the phase 2 and 3 studies comprised 4050 patients. The overall safety population for phase 3 studies included 3712 patients, of whom 183 comprised the safety population bacteremia subset. The overall mMITT population included 2585 patients, and the mMITT population bacteremia subset comprised 164 patients (ceftazidime-avibactam, $n=85$ [52.4\%]; comparator, $n=78$ [47.6\%]). The overall GNeME population included 1910 patients, and the GNeME population bacteremia subset comprised 101 patients (ceftazidime-avibactam, $n=54$ [53.5\%]; comparator, $n=47$ [46.5\%]). An overview of patient numbers included within each analysis set with the breakdown by treatment group is shown in Fig. 1.

\section{Patient Characteristics and Baseline Pathogens}

Baseline demographic characteristics for patients in the mMITT population (bacteremia subset) were generally similar between the ceftazidime-avibactam and comparator arms (Table 1). On average, patients in the bacteremia subset were slightly older (mean age $\sim 60$ years) than in the overall safety population (mean age $\sim 53$ years), and a larger proportion was female $(\sim 64 \%$ in the bacteremia subset versus $\sim 47 \%$ overall). The most common primary diagnoses were acute pyelonephritis and VAP (Table 1). Mean Acute Physiology and Chronic Health Evaluation (APACHE) II scores at baseline were higher in the NP/VAP population than in the cIAI population (Table 1); APACHE II score data were not collected for the patients with cUTI/ pyelonephritis in the RECAPTURE and REPRISE studies. For the indications where APACHE II scores were collected, mean (standard deviation [SD]) baseline APACHE II scores for the bacteremia safety subset were higher than for the overall safety population for both treatment groups (for example, in the ceftazidime-avibactam group and the cIAI indication, the mean [SD] APACHE II score for the bacteremia subset was 9.0 [5.9], whereas for the overall set it was $6.0[4.3])$.

The most frequently isolated pathogens in patients treated with ceftazidime-avibactam in the bacteremia subset (GNeME population) were Escherichia coli $(68.5 \%), \quad P$. aeruginosa (20.4\%), K. pneumoniae (18.5\%), and Enterobacter cloacae (7.4\%) (Table A2). Proportions of patients with these pathogens were similar in the overall set $(61.3 \%, 12.3 \%, 19.9 \%$, and $5.4 \%$, respectively). Ceftazidime-non-susceptible pathogens (minimum inhibitory concentration $\geq 8 \mu \mathrm{g} / \mathrm{l}$ for Enterobacterales and $\geq 16 \mu \mathrm{g} / \mathrm{l}$ for $P$. aeruginosa; or disk diffusion diameter $\leq 20 \mathrm{~mm}$ for Enterobacterales and $\leq 17 \mathrm{~mm}$ for $P$. aeruginosa), most frequently $E$. coli and K. pneumoniae, were identified in $30(29.7 \%)$ patients in the GNeME population bacteremia subset (Table A2). Ceftazidime-non-susceptible pathogens were similar in the overall set, identified in 604 (31.6\%) patients. Baseline 


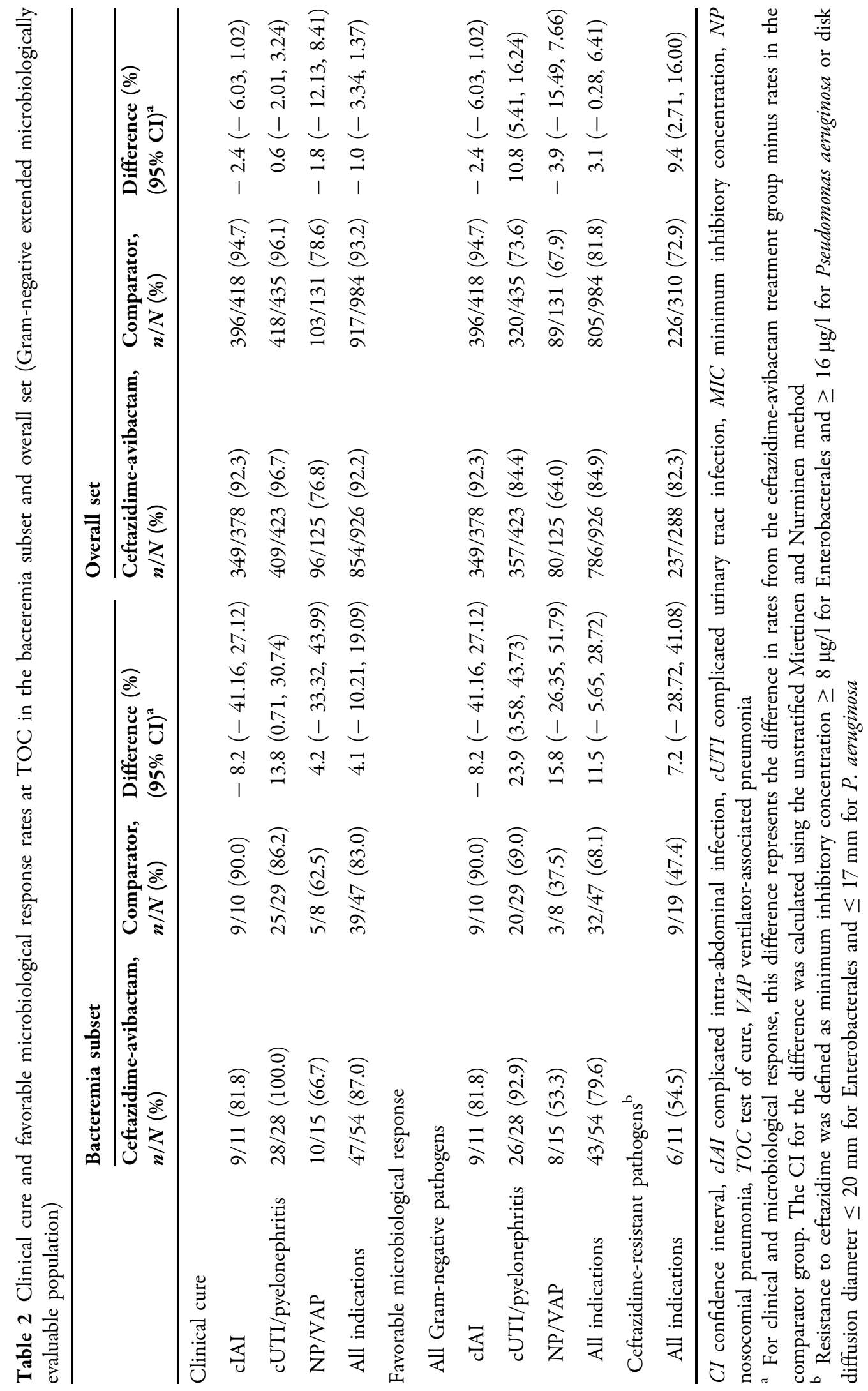


pathogens in the mMITT population were generally similar to those observed in the GNeME population (Table A3).

\section{Efficacy Assessments}

Efficacy results described here will focus on the GNeME population for the bacteremia subset and for the overall set. In the bacteremia subset, clinical cure rates at TOC for each of the four most commonly isolated pathogens were generally similar for ceftazidime-avibactam and comparators within each indication as well as combined across indications. Clinical cure rates for all indications combined were 47/54 (87.0\%) for ceftazidime-avibactam and 39/47 (83.0\%) for comparators and were generally similar to the overall set (Table 2). Clinical cure rates at TOC for each of the four most isolated pathogens in the bacteremia subset were generally similar between treatment groups, with numerical differences but wide-ranging CIs among individual pathogens, and were generally similar to the overall set (Table A4). Clinical cure rates for Enterobacterales were 44/50 $(88.0 \%)$ in the ceftazidime-avibactam group and $37 / 45(82.2 \%)$ in the comparator group. For $P$. aeruginosa, cure rates were $8 / 11(72.7 \%)$ in the ceftazidime-avibactam group and 5/6 (83.3\%) in the comparator group, in line with the overall set (Table A4). Similar results at TOC were observed for the mMITT population (Tables A5 and A6).

Favorable microbiological response rates at TOC in the GNeME bacteremia subset were generally similar for ceftazidime-avibactam and comparators within each indication and across all indications combined, considering the low denominators (Table 2). However, for the cUTI/ pyelonephritis indication, favorable microbiological response rates in the bacteremia subset were $26 / 28(92.9 \%)$ for ceftazidime-avibactam and 20/29 (69.0\%) for comparators and in the overall set were $357 / 423(84.4 \%)$ and $320 / 435$ (73.6\%), respectively (Table 2). For the 30 patients with ceftazidime-non-susceptible isolates, favorable microbiological response rates at TOC across all indications combined were $6 / 11$ $(54.5 \%)$ for ceftazidime-avibactam and 9/19
(47.4\%) for comparators (Table 2). Similar results were observed for the mMITT population (Table A5). Favorable per-pathogen microbiological response rates at TOC in the bacteremia subset were generally similar between treatment groups, with numerical differences and wideranging CIs among individual pathogens (Table A7). Of the four most commonly isolated pathogens at baseline, favorable microbiological response rates were, on average, higher for Enterobacterales than for $P$. aeruginosa in both the ceftazidime-avibactam and comparator groups (Table A7). Similar results were observed for the mMITT population (Table A8).

\section{Safety Assessments}

The median (range) duration of exposure to ceftazidime-avibactam was 7.0 (1-14) days in the bacteremia safety subset compared with 8.0 (1-21) days in the overall safety population.

Most AEs occurred in only one patient in either treatment group and were mild in intensity; no patterns were identified. The most common AEs were known adverse drug reactions to ceftazidime-avibactam [18] (Medical Dictionary for Regulatory Activities Preferred Terms): diarrhea (12/99 [12.1\%] for ceftazidimeavibactam) and nausea (9/99 [9.1\%] for ceftazidime-avibactam) (Table 3). In the safety population bacteremia subset, serious AEs were reported for $12 / 99(12.1 \%)$ and 8/84 (9.5\%) patients treated with ceftazidime-avibactam and comparators, respectively, compared with $176 / 2024(8.7 \%)$ and $145 / 2026$ (7.2\%), respectively, in the overall safety population. The frequency of diarrhea in patients treated with ceftazidime-avibactam was $12 / 99$ (12.1\%) in the bacteremia safety subset compared with $150 / 2024(7.4 \%)$ in the overall safety population.

In total, there were $3 / 99$ (3.0\%) deaths in patients in the bacteremia safety subset treated with ceftazidime-avibactam (Table A9); all were considered unrelated to study treatment. Of these, 2/99 (2.0\%) were reported as AEs. The remaining death was due to disease progression. In the comparator arm (bacteremia subset), there were $3 / 84(3.6 \%)$ deaths, one $(1.2 \%)$ of 


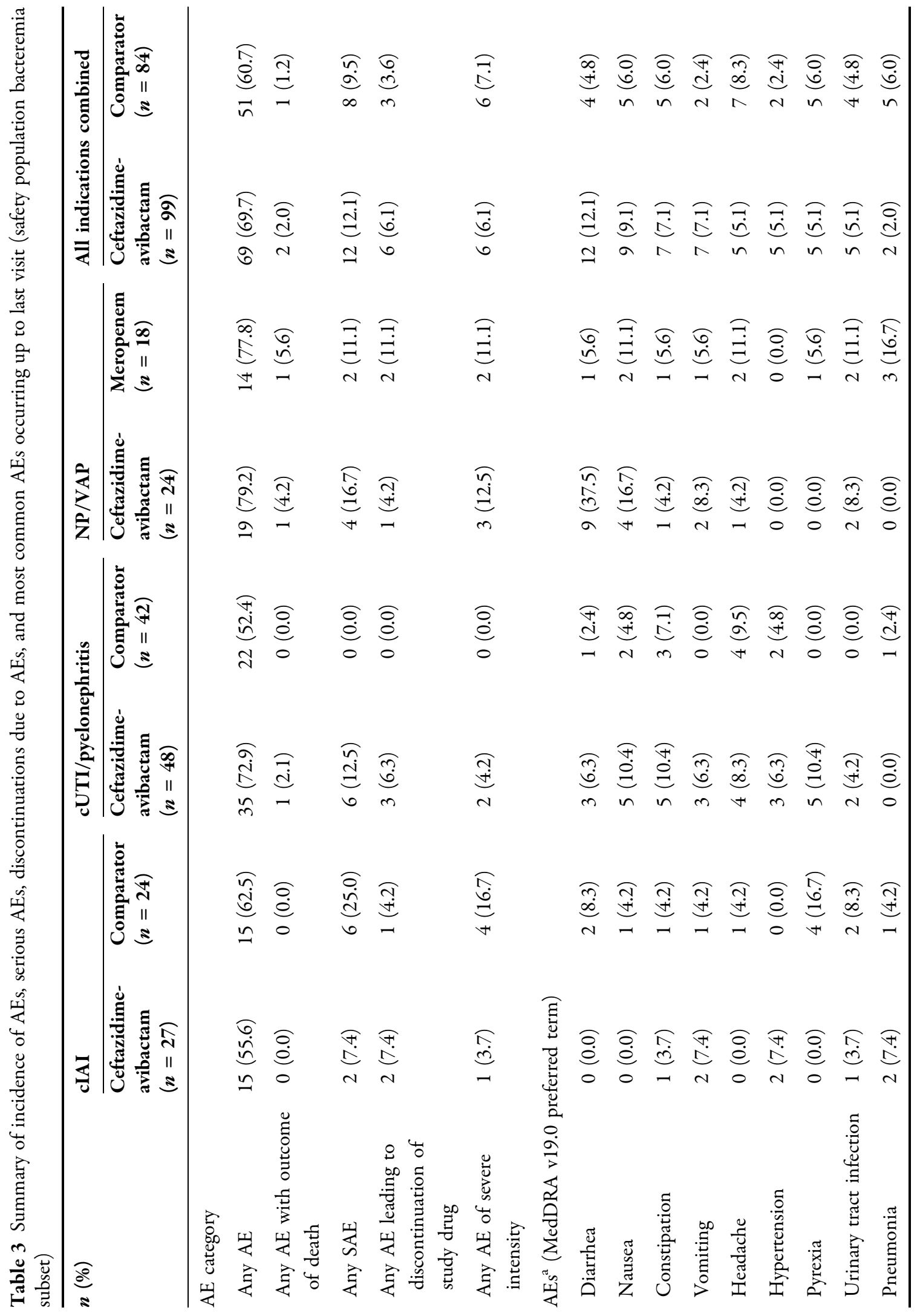




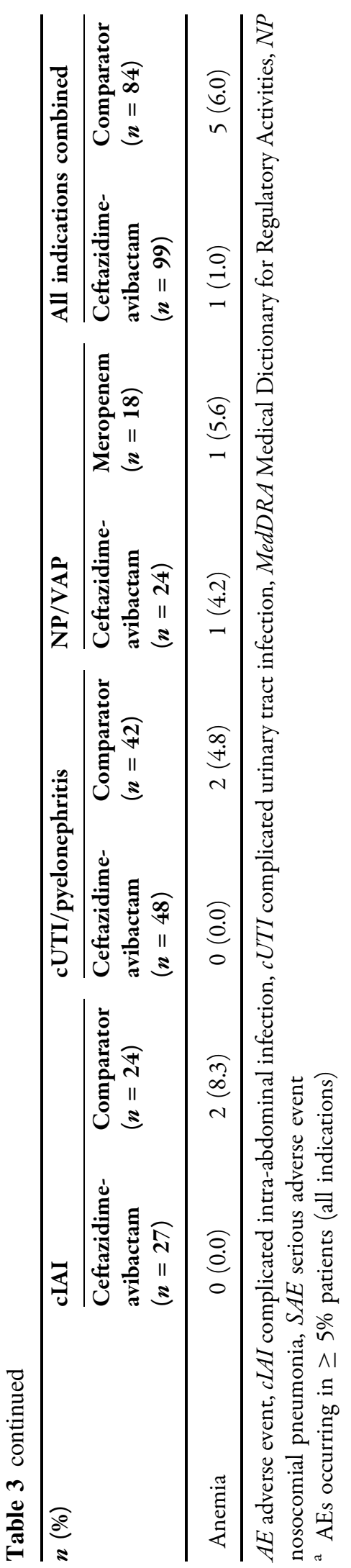

which was reported as an $\mathrm{AE}$ and two (2.4\%) as disease progression.

\section{DISCUSSION}

This descriptive analysis provides supportive evidence of the efficacy and safety of ceftazidime-avibactam in patients with Gramnegative bacteremia associated with cIAI, cUTI/ pyelonephritis, or NP/VAP across five phase 3 studies. Comparator treatments represented standard therapies in the respective indications and were generally carbapenems. As one of the two phase 2 studies used a lower dose of ceftazidime-avibactam than that used in the phase 3 program, and as it was not possible to programmatically confirm bacteremia at baseline due to different methods of data collection in the phase 2 versus phase 3 studies, the limited numbers of bacteremic patients from the two phase 2 studies were not included in the current bacteremia subset analyses. Of note, in Europe, the ceftazidime-avibactam label has recently been extended to include patients with bacteremia associated with cIAI, cUTI/ pyelonephritis, or NP/VAP $[17,18]$.

Favorable clinical and microbiological response rates at TOC in patients with Gramnegative bacteremia in the GNeME population were similar for ceftazidime-avibactam and comparators within each indication and across all indications combined, considering the low denominators. Across all indications combined, $87 \%$ of patients in the ceftazidime-avibactam group and $83 \%$ of those in the comparator group achieved clinical cure at TOC. Results in the mMITT population were largely consistent with those reported for the GNeME population. Of the four most commonly isolated bacteria at baseline (E. coli, K. pneumoniae, $P$. aeruginosa, and E. cloacae), favorable microbiological response rates were generally high for Enterobacterales infections and more variable for those caused by $P$. aeruginosa, for both ceftazidime-avibactam and comparator, consistent with observations from another recent analysis [23]. Of the 30 patients with ceftazidime-resistant pathogens, $54.5 \%$ of those treated with ceftazidime-avibactam and $47.4 \%$ of those 
treated with comparators had favorable microbiological response rates. However, the small patient numbers $(<5$ in each treatment group for almost all pathogens) limit interpretation of these results.

Multiple factors may affect the outcome in bacteremia, including the site of primary infection, species of the infecting organism, prompt initiation of antibiotic therapy, adequate source control, and host comorbidities $[6,24,25]$. This analysis included patients who all had secondary Gram-negative bacteremia associated with cIAI, cUTI/pyelonephritis, or NP/VAP. Despite the fact that, in general, patients in the bacteremia subset were more severely ill at baseline versus the overall set, the observed clinical and microbiological efficacy results for bacteremic patients treated with ceftazidime-avibactam showed favorable response rates that were broadly consistent with the respective overall analysis set for each of the indications as well as across all indications combined. Numerically, clinical cure rates were lower in patients with NP/VAP in the GNeME population bacteremia subset $(66.7 \%$ and $62.5 \%$ in the ceftazidime-avibactam and comparator groups, respectively) than for the other indications, in which rates ranged from 86.2 to $100 \%$. Differences in clinical outcomes between indications may, to an extent, reflect differences in baseline disease severity and comorbidities between cIAI, cUTI/pyelonephritis, and NP/VAP patient populations. However, the observed response rates by primary indication in patients with secondary Gram-negative bacteremia remained broadly consistent with those observed for the overall set in each of the respective indications, including NP/VAP, lending support to use of ceftazidime-avibactam in this patient population in clinical practice.

Several recent observational studies provide further supportive evidence for the efficacy of ceftazidime-avibactam in patients with Gramnegative bacteremia [26-28]. Similar to the $87 \%$ favorable clinical response rates in the bacteremia subset in the current analysis, a retrospective study in patients with carbapenemresistant K. pneumoniae bacteremia reported 85\% 30-day clinical success rate in patients treated with ceftazidime-avibactam, which was significantly higher than for carbapenem-based regimens: $48 \%$ (carbapenem plus aminoglycoside $[P=0.04]$ ); $40 \%$ (carbapenem plus colistin $[P=0.009]$ ); $37 \%$ (others; including monotherapy with aminoglycoside or colistin $[P=0.004])$ [28]. Similar findings have been reported in a meta-analysis of nine randomized controlled studies and three observational studies [29]. A recent retrospective study also identified ceftazidime-avibactam as the only independent predictor of survival among patients with bacteremia caused by carbapenemase-producing K. pneumoniae [30].

No new safety issues were identified for ceftazidime-avibactam. The safety profile of ceftazidime-avibactam in the bacteremia subset was in line with that observed for the pooled overall safety population [21], the established safety profile of ceftazidime alone, and the pattern of AEs expected for the patient population.

The most common AE for ceftazidime-avibactam was diarrhea, which is a known adverse drug reaction $[16,18]$. The frequency of diarrhea in the ceftazidime-avibactam group was higher in the bacteremia safety subset (12.1\%) than in the overall safety population (7.4\%). On detailed analysis, this difference was found not to be clinically relevant and may be explained by the small denominator and the increased age of the patients in the bacteremia subset, as a similar increase in frequency of diarrhea was seen in patients $>75$ years of age in the overall safety population [32/314 (10.2\%)].

A higher frequency of serious AEs was reported in the bacteremia subset than in the overall safety population; however, this was not accompanied by an increase in the rate of mortality or AEs with a fatal outcome, which is in line with the frequency observed in the overall safety population (2.0\%) [21]. With the caveat that this difference may be magnified by the smaller denominator, this can be explained by the difference in baseline condition of patients with Gram-negative bacteremia, who may be expected to be more unwell, as evidenced by higher baseline APACHE II scores in the patients with cIAI and NP/VAP, and to have associated symptoms such as tachycardia, fever, or sepsis. 


\section{CONCLUSION}

This exploratory analysis included patients with secondary Gram-negative bacteremia associated with cIAI, cUTI/pyelonephritis, or NP/VAP. As this subset comprised a limited number of patients, these analyses were descriptive and therefore must be interpreted with caution. Despite these limitations, these data support the efficacy and safety of ceftazidime-avibactam for the treatment of patients with Gram-negative bacteremia associated with cIAI, cUTI (including pyelonephritis), or NP/VAP.

\section{ACKNOWLEDGEMENTS}

The authors thank the patients, their families, and all investigators involved in the ceftazidime-avibactam clinical trial program.

Funding. This analysis was sponsored by Pfizer. Infectious Diseases and Therapy Rapid Service Fees were funded by the sponsor.

Medical Writing Assistance. Medical writing support was provided by Melanie More of Prime, Knutsford, Cheshire, UK, and was funded by Pfizer. Ultimate responsibility for opinions, conclusions, and data interpretation lies with the authors.

Authorship. All named authors meet the International Committee of Medical Journal Editors (ICMJE) criteria for authorship for this article, take responsibility for the integrity of the work as a whole, and have given their approval for this version to be published.

Author contributions. Margaret Tawadrous, Dalia Wajsbrot, Gregory G. Stone, and Paurus Irani designed and conceived the analysis. John E. Mazuski, Florian Wagenlehner, Antoni Torres, and Yehuda Carmeli performed the research in the original studies. John E. Mazuski, Florian Wagenlehner, Antoni Torres, Yehuda Carmeli, Joseph W. Chow, Dalia Wajsbrot, Gregory G. Stone, Paurus Irani, David Bharucha, Karen Cheng, and Margaret Tawadrous had roles in analysis and/or interpretation of the data. All authors were involved in drafting and/or critically revising the Article.

Disclosures. John E. Mazuski, Florian Wagenlehner, Antoni Torres, and Yehuda Carmeli received institutional research funding from AstraZeneca for conducting clinical studies included in this analysis. AstraZeneca's rights to ceftazidime-avibactam were acquired by Pfizer in December 2016. Ceftazidime-avibactam is being co-developed by Pfizer and AbbVie (following its acquisition of Allergan). Joseph W. Chow, Dalia Wajsbrot, Gregory G. Stone, Paurus Irani, Karen Cheng, and Margaret Tawadrous are employees of Pfizer and hold Pfizer shares/and or stock options. David Bharucha is an employee of AbbVie (following its acquisition of Allergan).

Compliance with ethics guidelines. This subset analysis was based on five previously reported phase 3 ceftazidime-avibactam clinical trials. Ethics approval and patient consent to participate were obtained for each of the previously reported trials, and all trials were conducted in accordance with the Helsinki Declaration of 1964 and its later amendments.

Data availability. The datasets generated during and/or analyzed during the current study are not publicly available. Upon request, and subject to review, Pfizer will provide the data that support the findings of this study. Subject to certain criteria, conditions, and exceptions, Pfizer may also provide access to the related individual anonymized participant data. See https://www.pfizer.com/science/clinical-trials/trial-data-and-results for more information.

Open Access. This article is licensed under a Creative Commons Attribution-NonCommercial 4.0 International License, which permits any non-commercial use, sharing, adaptation, distribution and reproduction in any medium or format, as long as you give appropriate credit to the original author(s) and the source, provide a link to the Creative Commons licence, and indicate if changes were made. The images or other third party material 
in this article are included in the article's Creative Commons licence, unless indicated otherwise in a credit line to the material. If material is not included in the article's Creative Commons licence and your intended use is not permitted by statutory regulation or exceeds the permitted use, you will need to obtain permission directly from the copyright holder. To view a copy of this licence, visit http:// creativecommons.org/licenses/by-nc/4.0/.

\section{REFERENCES}

1. Sligl W, Taylor G, Brindley PG. Five years of nosocomial gram-negative bacteremia in a general intensive care unit: epidemiology, antimicrobial susceptibility patterns, and outcomes. Int J Infect Dis. 2006;10(4):320-5.

2. Centers for Disease Control and Prevention. Antibiotic resistance threats in the United States, 2019. 2019. https://www.cdc.gov/drugresistance/ pdf/threats-report/2019-ar-threats-report-508.pdf. Accessed 29 January 2020

3. Vallés J, Alvarez-Lerma F, Palomar M, et al. Healthcare-associated bloodstream infections at admission to the ICU. Chest. 2011;139(4):810-5.

4. Bassetti M, Righi E, Carnelutti A. Bloodstream infections in the intensive care unit. Virulence. 2016;7(3):267-79.

5. Tansarli GS, Andreatos N, Pliakos EE, Mylonakis E. A systematic review and meta-analysis of antibiotic treatment duration for bacteremia due to Enterobacteriaceae. Antimicrob Agents Chemother. 2019;63(5):e02495-e2518.

6. van Duin D, Kaye KS, Neuner EA, Bonomo RA. Carbapenem-resistant Enterobacteriaceae: a review of treatment and outcomes. Diagn Microbiol Infect Dis. $2013 ; 75(2): 115-20$.

7. Falcone M, Bassetti M, Tiseo $G$, et al. Time to appropriate antibiotic therapy is a predictor of outcome in patients with bloodstream infection caused by KPC-producing Klebsiella pneumoniae. Crit Care. 2020;24(1):29.

8. Carmeli Y, Armstrong J, Laud PJ, et al. Ceftazidimeavibactam or best available therapy in patients with ceftazidime-resistant Enterobacteriaceae and Pseudomonas aeruginosa complicated urinary tract infections or complicated intra-abdominal infections (REPRISE): a randomised, pathogen-directed, phase 3 study. Lancet Infect Dis. 2016;16(6): 661-73.

9. Mazuski JE, Gasink LB, Armstrong J, et al. Efficacy and safety of ceftazidime-avibactam plus metronidazole versus meropenem in the treatment of complicated intra-abdominal infection: results from a randomized, controlled, double-blind, phase 3 program. Clin Infect Dis. 2016;62(11):1380-9.

10. Qin X, Tran BG, Kim MJ, et al. A randomised, double-blind, phase 3 study comparing the efficacy and safety of ceftazidime/avibactam plus metronidazole versus meropenem for complicated intraabdominal infections in hospitalised adults in Asia. Int J Antimicrob Agents. 2017;49(5):579-88.

11. Torres A, Zhong N, Pachl J, et al. Ceftazidime-avibactam versus meropenem in nosocomial pneumonia, including ventilator-associated pneumonia (REPROVE): a randomised, double-blind, phase 3 non-inferiority trial. Lancet Infect Dis. 2018;18(3): 285-95.

12. Wagenlehner FM, Sobel JD, Newell P, et al. Ceftazidime-avibactam versus doripenem for the treatment of complicated urinary tract infections, including acute pyelonephritis: RECAPTURE, a phase 3 randomized trial program. Clin Infect Dis. 2016;63(6):754-62.

13. Stone GG, Bradford PA, Yates K, Newell P. In vitro activity of ceftazidime/avibactam against urinary isolates from patients in a Phase 3 clinical trial programme for the treatment of complicated urinary tract infections. J Antimicrob Chemother. 2017;72(5):1396-9.

14. Stone GG, Bradford PA, Newell P, Wardman A. In vitro activity of ceftazidime-avibactam against isolates in a phase 3 open-label clinical trial for complicated intra-abdominal and urinary tract infections caused by ceftazidime-nonsusceptible gram-negative pathogens. Antimicrob Agents Chemother. 2017;61(2):e01820-e1916.

15. Stone GG, Newell P, Bradford PA. In vitro activity of ceftazidime-avibactam against isolates from patients in a phase 3 clinical trial for treatment of complicated intra-abdominal infections. Antimicrob Agents Chemother. 2018;62(7):e02584-e2617.

16. Allergan. AVYCAZ (ceftazidime and avibactam) for injection, for intravenous use. 2020. https://www. allergan.com/assets/pdf/avycaz_pi. Accessed 19 February 2021

17. European Medicines Agency. Zavicefta. Opinion. 2020. https://www.ema.europa.eu/en/medicines/ human/summaries-opinion/zavicefta-0. Accessed 9 November 2020 
18. Pfizer. Summary of Product Characteristics: Zavicefta $2 \mathrm{~g} / 0.5 \mathrm{~g}$ powder for concentrate for solution for infusion. 2021. https://www.ema.europa.eu/ documents/product-information/zavicefta-eparproduct-information_en.pdf. Accessed 19 February 2021

19. Lucasti C, Popescu I, Ramesh MK, Lipka J, Sable C. Comparative study of the efficacy and safety of ceftazidime/avibactam plus metronidazole versus meropenem in the treatment of complicated intraabdominal infections in hospitalized adults: results of a randomized, double-blind, Phase II trial. J Antimicrob Chemother. 2013;68(5):1183-92.

20. Vazquez JA, Gonzalez Patzan LD, Stricklin D, et al. Efficacy and safety of ceftazidime-avibactam versus imipenem-cilastatin in the treatment of complicated urinary tract infections, including acute pyelonephritis, in hospitalized adults: results of a prospective, investigator-blinded, randomized study. Curr Med Res Opin. 2012;28(12):1921-31.

21. Cheng K, Newell P, Chow JW, et al. Safety profile of ceftazidime-avibactam: pooled data from the adult Phase II and Phase III clinical trial programme. Drug Saf. 2020;43(8):751-66.

22. Miettinen $\mathrm{O}$, Nurminen M. Comparative analysis of two rates. Stat Med. 1985;4(2):213-26.

23. Nichols WW, Stone GG, Newell P, et al. Ceftazidime-avibactam susceptibility breakpoints against Enterobacteriaceae and Pseudomonas aeruginosa. Antimicrob Agents Chemother. 2018;62: e02590-e2617.
24. Blot S. Setting the baseline to fight gram-negative bacteraemia: the necessity of epidemiological insights. Infect Dis (Lon, Engl). 2019;51(1):23-5.

25. Ho J, Tambyah PA, Paterson DL. Multiresistant gram-negative infections: a global perspective. Curr Opin Infect Dis. 2010;23(6):546-53.

26. Wu G, Abraham T, Lee S. Ceftazidime-avibactam for treatment of carbapenem-resistant Enterobacteriaceae bacteremia. Clin Infect Dis. 2016;63(8): 1147-8.

27. Castón JJ, Lacort-Peralta I, Martin-Davila P, et al. Clinical efficacy of ceftazidime/avibactam versus other active agents for the treatment of bacteremia due to carbapenemase-producing Enterobacteriaceae in hematologic patients. Int J Infect Dis. 2017;59:118-23.

28. Shields RK, Nguyen MH, Chen L, et al. Ceftazidimeavibactam is superior to other treatment regimens against carbapenem-resistant Klebsiella pneumoniae bacteremia. Antimicrob Agents Chemother. 2017;61(8):e00883-e917.

29. Zhong H, Zhao XY, Zhang ZL, et al. Evaluation of the efficacy and safety of ceftazidime/avibactam in the treatment of gram-negative bacterial infections: a systematic review and meta-analysis. Int $\mathrm{J}$ Antimicrob Agents. 2018;52(4):443-50.

30. Tumbarello M, Trecarichi EM, Corona A, et al. Efficacy of ceftazidime-avibactam salvage therapy in patients with infections caused by Klebsiella pneumoniae carbapenemase-producing K. pneumoniae. Clin Infect Dis. 2019;68(3):355-64. 